\title{
Perceived Size Change Induced by Nonvisual Signals in Darkness: The Relative Contribution of Vergence and Proprioception
}

\author{
Irene Sperandio, ${ }^{1,2}$ Shaleeza Kaderali, ${ }^{3}$ Philippe A. Chouinard, ${ }^{1}$ Jared Frey, ${ }^{4}$ and Melvyn A. Goodale ${ }^{1}$ \\ ${ }^{1}$ Brain and Mind Institute, and Department of Psychology, University of Western Ontario, London, Ontario N6A 5B8, Canada, ${ }^{2}$ School of Psychology, \\ University of East Anglia, Norwich NR4 7TJ, United Kingdom, ${ }^{3}$ McGill Vision Research, Department of Ophthalmology, McGill University, Montreal, \\ Quebec H3A 1A1, Canada, and ${ }^{4}$ Department of Bioengineering, University of California, Los Angeles, Los Angeles, California 90095
}

\begin{abstract}
Most of the time, the human visual system computes perceived size by scaling the size of an object on the retina with its perceived distance. There are instances, however, in which size- distance scaling is not based on visual inputs but on extraretinal cues. In the Taylor illusion, the perceived afterimage that is projected on an observer's hand will change in size depending on how far the limb is positioned from the eyes- even in complete darkness. In the dark, distance cues might derive from hand position signals either by an efference copy of the motor command to the moving hand or by proprioceptive input. Alternatively, there have been reports that vergence signals from the eyes might also be important. We performed a series of behavioral and eye-tracking experiments to tease apart how these different sources of distance information contribute to the Taylor illusion. We demonstrate that, with no visual information, perceived size changes mainly as a function of the vergence angle of the eyes, underscoring its importance in size-distance scaling. Interestingly, the strength of this relationship decreased when a mismatch between vergence and proprioception was introduced, indicating that proprioceptive feedback from the arm also affected size perception. By using afterimages, we provide strong evidence that the human visual system can benefit from sensory signals that originate from the hand when visual information about distance is unavailable.
\end{abstract}

\section{Introduction}

It has been repeatedly observed that the perceived size of an afterimage- the "imprinted" image on the retina after the brief exposure of a bright source of light-appears larger when "projected" on surfaces farther from the eyes, and smaller when projected nearer. This simple experiment with afterimages, formally known as Emmert's law (Emmert, 1881), is a demonstration of size constancy: the ability to achieve a stable experience of perceived size despite distance variations as a result of a "recalibration" of retinal image size with perceived distance (Andrews, 1964; Morgan, 1992).

In complete darkness, with no visual cues to indicate alterations in distance, changes in perceived afterimage size can still occur by means of extraretinal cues. For example, an afterimage appears bigger as the observer's hand (Taylor, 1941; Carey and

\footnotetext{
Received March 4, 2013; revised Sept. 12, 2013; accepted Sept. 17, 2013.

Author contributions: I.S., P.A.C., and M.A.G. designed research; I.S. and S.K. performed research; I.S., S.K., and J.F. analyzed data; I.S., S.K., P.A.C., and M.A.G. wrote the paper.

This research was supported by a postdoctoral fellowship from the Ontario Ministry of Research and Innovation to I.S., a postdoctoral fellowship award from the 0 ntario Mental Health Foundation to P.A.C., and an operating grant from Natural Sciences and Engineering Research Council of Canada to M.A.G. The authors thank J. Ladich for constructing the light stimulus; D. Vendramini for building the equipment; H. Yang for technical support; and M. Barnett-Cowan and R.L. Whitwell for statistical advice.

The authors declare no competing financial interests.

Correspondence should be addressed to either Irene Sperandio or Melvyn A. Goodale, Brain and Mind Institute and the Department of Psychology, University of Western Ontario, 1151 Richmond Street, London, ON N6A 5B8, Canada.E-mail: i.sperandio@uea.ac.uk or mgoodale@uwo.ca.

DOI:10.1523/JNEUROSCI.0977-13.2013

Copyright $\odot 2013$ the authors $\quad 0270-6474 / 13 / 3316915-09 \$ 15.00 / 0$
}

Allan, 1996) or head (Gregory et al., 1959) moves away from the light that had initially generated the afterimage. This demonstration of size-distance scaling in darkness is known as the "Taylor illusion" (Carey and Allan, 1996). The Taylor illusion is an intriguing instance of multimodal integration in which vision is not directly modulated by new retinal inputs but by other sensory modalities. At first glance, it would seem reasonable to deduce that distance information contributing to the Taylor illusion can be derived from signals related to arm movement, such as efference copy or proprioceptive signals (Davies, 1973; Carey and Allan, 1996; Bross, 2000; Ramsay et al., 2007; Hogendoorn et al., 2009). Nevertheless, there have been claims that this phenomenon is driven solely by vergence (Taylor, 1941; Mon-Williams et al., 1997). These claims warrant further examination since most of the previous studies have not accounted for vergence registration in the dark and/or psychophysical measurement of the illusory effects (for review of the methodological limitations in previous research, see Ramsay et al., 2007).

By continuously monitoring eye positioning and by using a perceptual judgment task, as described by Ramsay et al. (2007), we performed three experiments on the Taylor illusion. In experiment 1 , we tested the illusion under active and passive movement conditions while participants fixated their gaze at different locations: namely, on their hand, at a fixed location away from their hand, or without a particular fixation. Results from this experiment revealed that an efference copy did not play a major role, given that passive and active movements affected the illusion equally, and that fixating on an LED attached to the moving hand 
A

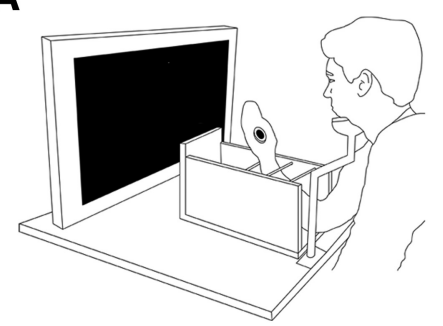

B

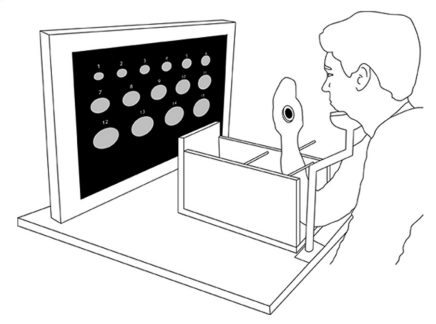

C

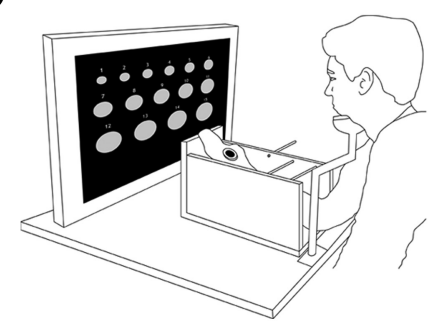

Figure 1. Layout of the experimental setup. $\boldsymbol{A}$, An afterimage was generated in darkness through a hand-held ring of lights placed in the palms of the participants at $25 \mathrm{~cm}$ from the eyes. $\boldsymbol{B}$, In the toward condition, the arm was displaced from the starting position to the near dowel at $12.5 \mathrm{~cm}$ from the eyes. $\boldsymbol{C}$, In the away condition, the arm was displaced from the starting position to the far wall of the box at $50 \mathrm{~cm}$ from the eyes. Participants performed a size-matching task upon execution of the movement to judge the perceived size of the afterimage. The monitor displaying the reference stimuli for the size-matching task was turned off during the induction of the afterimage.

boosted the illusory effect-confirming that vergence is quite relevant. In experiment 2, we recorded eye movements to further investigate the role of vergence in the illusion and found a tight relationship between vergence angle and perceived size. In experiment 3, we examined the relative contributions of vergence and proprioceptive cues by using a fixation point that moved either in the same or in the opposite direction as the hand. In this experiment, we found a weaker relationship between vergence and perceived size on incongruent trials. We conclude that under restricted viewing conditions the visual system relies on multimodal signals to gather information about distance and make inferences about object size.

\section{Materials and Methods}

Overview. In the Taylor illusion, a bright white light is flashed on the hand while the participant is placed in an otherwise totally dark room. This flash of light creates a positive afterimage of a fixed size on the retina, yet as the participant moves his/her hand closer to the eyes the perceived afterimage gets smaller. Conversely, the perceived afterimage gets bigger as the participant moves his/her hand farther away from the eyes. To carry out this illusion in complete darkness, we constructed a glove that had of a ring of LEDs that could be worn on the palm of the hand. This ring of lights on the glove could be controlled by a computer. We also constructed an apparatus-shaped as a box-in which the participants placed their forearm (Fig. 1). Using this apparatus, we could control arm positioning in complete darkness such that the arm could be displaced from a central position (Fig. $1 A$ ) to another position that was either closer to the eyes (Fig. 1B) or farther away from the eyes (Fig. 1C). During the experiments, we also manipulated vergence by controlling a red fixation light. This fixation light could also be placed at various locations. To acquire measures of size perception, we presented at the end of each trial a series of gray-colored circles on a computer monitor (Fig. 1). These circles varied in size, and participants were required to indicate which one they thought best matched the size of the afterimage that they saw. Eye movements were also monitored in total darkness using an infrared tracking device. The rest of the Materials and Methods will describe in more detail these devices and the procedures that we used in each of our experiments.

Participants. In total, 28 healthy right-handed participants (12 males), ranging in age from 18 to 35 years, participated in three experiments, with some of the participants taking part in more than one experiment. Participants had normal or corrected-to-normal visual acuity. Nineteen participants ( 6 males; age range, $18-21$ years; mean age, 18.89 years) participated in experiment 1 , which tested the contribution of efference copy to the Taylor illusion. Six participants well trained in psychophysical testing (three males; age range, 21-35 years; mean age, 27.16 years) took part in experiment 2 , in which eye movements were recorded during the Taylor illusion. Finally, six participants (two males; age range 21-34 years; mean age, 30.5 years) performed experiment 3 , in which a mismatch between proprioception and vergence signals was induced, and eye movements were recorded. Each participant was required to fill out the 10-item version of the Edinburgh handedness questionnaire (Oldfield, 1971). Written consent was obtained before testing. All methods were compliant with the rules and regulations of the University of Western Ontario Ethics Review Board.

Devices. The stimuli and the psychophysical experiments were programmed in E-Prime (Psychology Software Tools) on a Dell Pws760 computer using the Windows XP operating system. Participants sat on a height-adjustable chair in front of a 32 inch NEC multisynch LCD monitor $(1024 \times 768$ pixel resolution $)$ with their head placed on a chin rest 57 $\mathrm{cm}$ from the monitor. To control arm movements during the experiments, we used an apparatus that consisted of a wooden box with three adjustable wooden dowels (Fig. 1). Participants placed their right arm inside the apparatus between two of the dowels, which marked the starting position at $25 \mathrm{~cm}$ from the eyes. The third dowel was placed at 12.5 $\mathrm{cm}$ from the eyes to mark the "toward" position, which was exactly half the distance from the starting position. The far wall of the box was placed at a viewing distance of $50 \mathrm{~cm}$, which was exactly double the distance from the starting position, to mark the "away" position. To minimize the appearance of the hand, participants wore a black glove and a $5 \mathrm{~cm}$ ring of white LED lights $\left(360^{\circ}\right.$; Super Bright LEDs Inc.), with a luminance of $2686 \mathrm{~cd} / \mathrm{m}^{2}$, attached to the palm of the glove to deliver the flash of light (Fig. 1A). The inducing stimulus light subtended $11.42^{\circ}$ of visual angle. A small red LED light was placed in the middle of the inducing stimulus light and used as a fixation point in some trials. Both inducing and fixation lights were controlled through E-Prime via a parallel port. To acquire measures of size perception, we presented at the end of each trial a series of gray-colored circles on a computer monitor (Ramsay et al., 2007). Specifically, 22 gray circles representing each point on a size rating scale numbered from 1 to 22 were presented on the LCD screen (luminance, $\left.36.93 \mathrm{~cd} / \mathrm{m}^{2}\right)$. The circles ranged in diameter from $1.5 \mathrm{~cm}\left(1.5^{\circ}\right.$ of visual angle) to $12 \mathrm{~cm}\left(12^{\circ}\right.$ of visual angle), increasing in $0.5 \mathrm{~cm}$ steps. By applying Emmert's law (see Eq. 3), we predicted that the perceived size of the afterimage would be $2.5 \mathrm{~cm}$ in diameter when viewed at a distance of $12.5 \mathrm{~cm}$ (i.e., the toward position), $5 \mathrm{~cm}$ in diameter at a distance of $25 \mathrm{~cm}$ (i.e., the starting position), and $10 \mathrm{~cm}$ in diameter at a distance of $50 \mathrm{~cm}$ (i.e., the away position). Thus, the range of our rating scale exceeded the smaller and larger theoretical sizes of the afterimage with perfect sizedistance scaling. In experiments 2 and 3, during which eye movements were recorded, participants wore a head-mounted eye-tracking system (Eyelink II, SR Research) that registered the position of both eyes at a sampling rate of $250 \mathrm{~Hz}$. The eye tracker was calibrated with a 9-point calibration display at the beginning of each block.

General procedure. Participants spent an initial $5 \mathrm{~min}$ in a completely darkened room to allow for retinal adaptation to the darkness. At the beginning of each trial, participants placed their right arm between the two dowels at the starting position at a viewing distance of $25 \mathrm{~cm}$ (Fig. $1 A$ ) and received auditory instructions (i.e., toward or away) about the direction of the movement to be executed. A vivid positive afterimage was then generated by a high-intensity flash of $150 \mathrm{~ms}$ from the handheld ring of LED lights. This afterimage lasted for $8-10 \mathrm{~s}$ on average. Following the induction of the afterimage, participants were instructed to slowly move their arm to the cued direction, after the removal of either 
dowel by the experimenter. In half of the trials, the hand was moved from the starting position to the closest dowel placed at a viewing distance of $12.5 \mathrm{~cm}$ (toward position; Fig. $1 B$ ). In the other half of the trials, the hand was moved from the starting position to the far wall of the box located at a viewing distance of $50 \mathrm{~cm}$ (away position; Fig. 1C). When performing hand movements, participants were instructed to rotate their forearm without lifting their elbow. This manipulation did not deform the perceived shape of the afterimage that was persistently reported as circular. On each trial, participants were instructed to register the perceived size of the afterimage at the end position and match it to one of the numbered circles presented on the LCD monitor. After the size-matching task and between each presentation of the stimulus, participants were instructed to close their eyes to prevent any incident light induced by the experimenter activity. Trials were initiated once the previous afterimage disappeared. Participants were also given four practice trials before testing began.

Procedures for experiment 1: the role of efference copy. The experiment consisted of 28 trials in total with two repetitions for every condition, as described below. Conditions differed depending on the type of arm movements being made and the location of eye fixation during the execution of these movements. Four control judgments were also collected without any movement of the arm to provide measurements of baseline.

To study the effects of efference copy in size perception, trials were divided into two blocks: one block of active movements and one block of passive movements (Carey and Allan, 1996; Bross, 2000). The order of these two blocks was counterbalanced across participants. The logic was to see whether or not in the active condition-in which participants are actively moving their hand-the corollary discharge of the motor command to the hand might influence the perceived size of afterimages. Under passive conditions, there should be no motor commands to the hand, given that the experimenter, not the participant, is moving the hand. Therefore, the main difference between active and passive conditions was efference copy (i.e., feedforward motor command) that was available during active movements only (Carey and Allan, 1996). The order of presentation of the two blocks was counterbalanced across participants. In the active condition, participants moved their hand themselves, cued by auditory instructions. In the passive condition, the experimenter, cued by auditory instructions, moved the participant's hand. In this condition, participants were completely unaware in which direction the experimenter would move their arm from trial to trial, and were instructed to keep their arm relaxed and refrain from hindering any movement of their limb. To study the effects of vergence on size perception, three different types of fixation conditions were tested for every direction of hand movement, as follows: (1) "central fixation," whereby the small red fixation light was turned on in the center of the hand-held ring after the presentation of the inducing stimulus light and participants were instructed to keep their eyes fixated on the small light and follow it as the hand was moved; (2) "no fixation," whereby only the inducing stimulus light was presented without any fixation cue ever being provided, and; (3) "lateral fixation," whereby the fixation light was presented next to the hand at $14.8^{\circ}$ eccentricity from the body's midline $(25 \mathrm{~cm}$ from the eyes), and participants were instructed to fixate on this stationary light immediately after the generation of the afterimage while the hand was moving. The order of these types of fixation was randomly determined for each of the "active" and "passive" blocks. In short, participants completed 24 experimental trials (i.e., 2 kinds of movement $\times 2$ hand directions $\times 3$ fixation types $\times 2$ repetitions) and 4 control trials (baseline).

Procedures for experiment 2: the role of vergence and proprioception. The protocol was similar to that of experiment 1, with the following two exceptions: (1) participants always performed active movements; and (2) vergence eye movements were recorded using a head-mounted eye tracker to ensure participants were, in fact, fixating appropriately, as well as to investigate further the relationship between vergence and perceived size of the afterimage. The experiment consisted of 48 trials in total (i.e., 2 hand directions $\times 3$ fixation types $\times 8$ repetitions). Trials were presented in a random fashion.

Procedures for experiment 3: mismatch between vergence and proprioception. To investigate the effect of vergence-proprioceptive mismatch
(Ramsay et al., 2007), participants were tested with congruent and incongruent trials. In the congruent condition, the fixation light was moved by the experimenter in the same direction as the observer's hand. In the incongruent condition, the fixation light was moved by the experimenter in the opposite direction to the observer's hand. Participants were asked to follow the fixation light with their eyes and to move their hand according to the cued direction. Eye movements were recorded for all participants. The experiment consisted of 32 trials in total (i.e., 2 hand directions $\times 2$ conditions $\times 8$ repetitions). Trials were presented in a random fashion.

Data analyses. For the purposes of statistical analysis, the self-reported data of perceived size were first normalized to allow meaningful comparison between conditions and provide measures of individual susceptibility to the illusion. This "susceptibility index" was calculated as follows: (perceived size of the afterimage at the away position - perceived size of the afterimage at the near position)/the sum of the perceived size of the afterimages at each of these two positions (Chouinard et al., 2013). To ensure that the illusion had been induced, a $t$ test against 0 was performed on the mean susceptibility indices for each condition. A repeatedmeasures ANOVA was also performed on these values with hand direction (toward vs away), movement type (active vs passive), and fixation type (central fixation vs no fixation vs lateral fixation) as main factors. All results were corrected for multiple comparisons using the Bonferroni method (i.e., $p_{\text {corrected }}=P_{\text {uncorrected }} \times$ the number of comparisons). For experiments 2 and 3, vergence was determined from eye position in a head-centered reference frame with saccades removed, in a manner similar to that described in a recent study by Frey and Ringach (2011). To measure the change in vergence after limb movement, the mean vergence angle at the end of the trial was subtracted from the mean vergence angle at the beginning of the trial. These two mean angles were determined by averaging vergence across $100 \mathrm{~ms}$ windows ( 25 data points). In this way, convergence eye movements (i.e., simultaneous inward rotation of both eyes to fixate at a near distance) were associated with positive values, while divergence eye movements (i.e., simultaneous outward rotation of both eyes to fixate at a far distance) were associated with negative values. These values were then correlated with the subjective ratings of the perceived afterimage size. Pearson correlation coefficients $(r)$ were calculated, and significance from these correlation coefficients was established using one-tailed criteria. This was deemed to be appropriate given that we already know on the basis of Emmert's law that perceived afterimage size increases with viewing distance. More importantly, we were interested in testing the hypothesis that changes in perceived size would always be completely predicted by changes in vergence (Taylor, 1941; Mon-Williams et al., 1997). A repeated-measures ANOVA with fixation type (central fixation vs no fixation vs lateral fixation) as a main factor (experiment 2) or a paired $t$ test (experiment 3 ) was performed on the correlation coefficients. Post hoc pairwise comparisons were adjusted using the Bonferroni correction. Two-tailed criteria were used for all the pairwise comparisons.

Additional analyses based on maximum likelihood estimation. To measure the relative contribution of vergence and proprioceptive cues to the Taylor illusion, the maximum likelihood estimation (MLE) principle was applied to estimates of distance for the no-fixation condition of experiment 2. Previous research has demonstrated that human multisensory perception of depth, height, size, and spatial location follows predictions based on the MLE model (Landy et al., 1995; Ernst and Banks, 2002; Gepshtein and Banks, 2003; Alais and Burr, 2004). According to this model, vergence and proprioceptive estimates are optimally combined to yield the lowest variance in an integrated (multimodal) estimate. By using the MLE rule, distance estimates from vergence and proprioceptive signals are weighted by their reliability such that the signal with lower variance (i.e., higher reliability) is given more weight than the signal with higher variance (i.e., lower reliability). The statistical strategy for cue combination is a weighted linear summation of each independent estimate:

$$
\hat{S}_{V P}=w_{V} \hat{S}_{V}+w_{P} \hat{S}_{P}
$$


where $\hat{S}_{V}, \hat{S}_{P}$, and $\hat{S}_{V P}$ are the vergence, proprioceptive, and combined estimates, respectively. The $w_{V}$ and $w_{P}$ are the unimodal weights given by the following:

$$
\begin{aligned}
& w_{V}=\frac{1 / \sigma V^{2}}{1 / \sigma V^{2}+1 / \sigma P^{2}} \\
& w_{P}=\frac{1 / \sigma P^{2}}{1 / \sigma V^{2}+1 / \sigma P^{2}}
\end{aligned}
$$

where $\sigma_{V}$ and $\sigma_{P}$ are SDs associated with vergence and proprioceptive estimates of distance.

Estimates of distance specified by vergence were computed from the vergence angle registered at the end of the movement. Estimates of distance specified by proprioception corresponded to the final location of the hand after the movement, namely $12.5 \mathrm{~cm}$ for toward trials and $50 \mathrm{~cm}$ for away trials. Finally, combined estimates of distance were obtained by applying Emmert's law (Emmert, 1881), as follows:

$$
d=\frac{s}{\tan \theta},
$$

where $d$ is the perceived distance, $s$ is the perceived size of the afterimage, and $\theta$ is the visual angle subtended by the afterimage. Vergence and proprioceptive weights were derived from the discrepancy of the unimodal estimates from the combined estimates of distance (Tresilian and Mon-Williams, 2000).

\section{Results}

Experiment 1: the role of efference copy In this experiment, we tested the Taylor illusion under active and passive movement conditions while participants fixated their gaze at different locations; namely, on their hand, at a fixed location away from their hand, or without a particular fixation position. Perceptual judgments indicated that the afterimage was experienced on every trial, and, hence, the movement of the hand did not disrupt the perception of the afterimage even when the line of sight did not coincide with the proprioceptive location of the hand (i.e., "lateral fixation" condition). Overall, our participants were susceptible to the Taylor illusion: when the hand was moved to the toward position, the size of the afterimage was perceived as smaller than its perceived size at the starting position, whereas when the hand was moved to the away position, the size of the afterimage was perceived as larger. A repeated-measures ANOVA on size judgments revealed a main effect of hand direction (toward vs away: $\left.F_{(1,18)}=66.37, p<0.0001\right)$. When asked to judge the size of the afterimage in the original position without any movement of the hand, participants were quite accurate in reporting the real size of the hand-held ring, which corresponded to a size rating of 8 (baseline: mean $=8.08, S D=1.1$; Fig. $2 A$, horizontal line). A $t$ test against 8 demonstrated that the perceived size of the hand-held ring was not statistically different from its actual size $\left(t_{(18)}=0.131, p=0.897\right)$.

To investigate the contribution of efference copy to the illusion and its possible interaction with vergence, we compared the two experimental conditions in which limb movements were made actively or passively. A two-way ANOVA was performed on

\section{Mean Raw Measurements for Active and Passive Conditions}

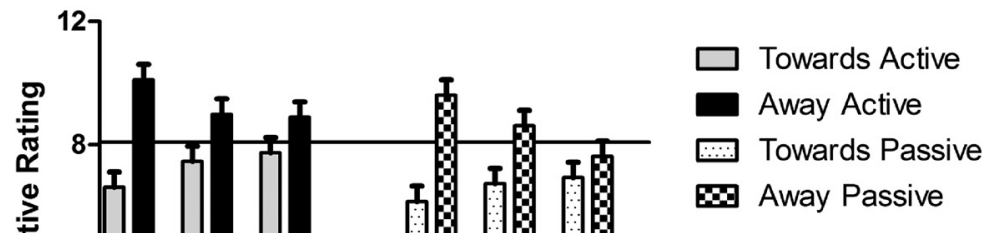

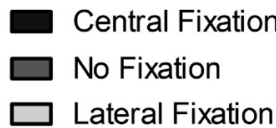

\section{Fixation Type}

Figure 2. Results of experiment 1. A, Mean size ratings as a function of condition (active/passive) and direction of movement (toward/away). The solid line designates the perceived size of the afterimage at the starting position (baseline). $\boldsymbol{B}$, Normalized effects of the Taylor illusion with data for active and passive conditions collapsed together. Error bars in the figure indicate withinsubject 95\% confidence intervals (WSCl; Loftus and Masson, 1994). Asterisks $\left(^{*}\right)$ denote significance after Bonferroni correction.

the normalized indices of illusion susceptibility (i.e., far - near/ far + near) with fixation type (central fixation vs no fixation vs lateral fixation) and movement type (active vs passive) as main factors. There was a main effect of fixation type $\left(F_{(2,36)}=14.591, p<\right.$ $0.0001)$, but neither the main effect of movement type nor its interaction with fixation were significant $\left(F_{(1,18)}=0.041, p=0.843\right.$; $F_{(2,36)}=0.671, p=0.518$, respectively). In other words, there were statistical differences between the fixation conditions but not between active and passive movement types, indicating that motor commands for limb movements had little or no contribution to the illusion. Data for active versus passive movements were therefore collapsed and further analyzed (Fig. $2 B$ ). A $t$ test against 0 was performed on the susceptibility index for the three fixation conditions to verify the occurrence of the Taylor illusion. This analysis confirmed that the illusion was indeed experienced by our participants under all tested conditions ( $p_{\text {corrected }}<0.01$ ). Post hoc pairwise comparisons showed significant differences between central (mean $=$ $0.21, \mathrm{SD}=0.13$ ) and no-fixation (mean $=0.11, \mathrm{SD}=0.09)$ conditions $\left(p_{\text {corrected }}=0.015\right)$, as well as central and lateral fixation $($ mean $=0.06, \mathrm{SD}=0.07)$ conditions $\left(p_{\text {corrected }}=0.0002\right)$. These results seem to suggest that vergence had the strongest effect on the perceived size of the afterimage. However, proprioceptive information seemed to play a small role in the generation of the illusion, 
A

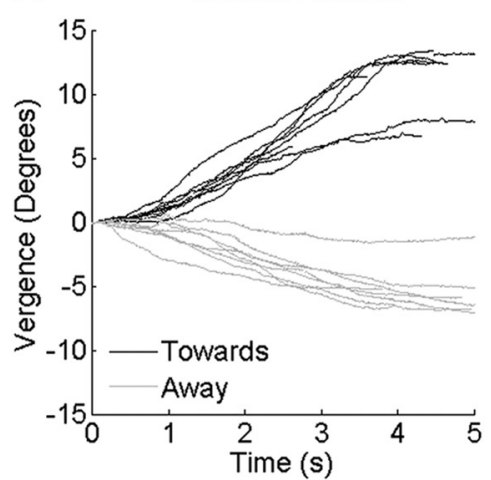

B

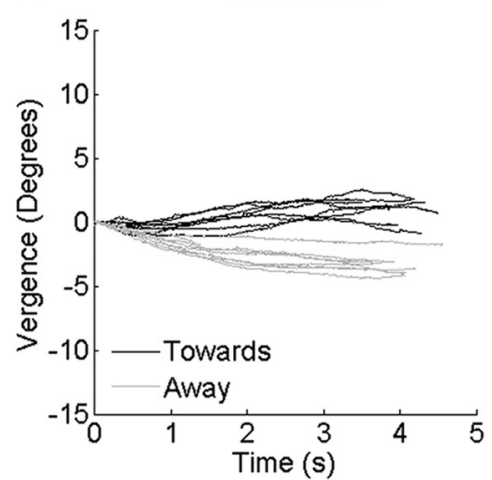

C



Figure 3. Examples of vergence signals in one representative participant. Vergence eye movements were registered by a head-mounted eye tracker. $\boldsymbol{A}-\boldsymbol{C}$, Each panel depicts vergence responses typically observed during central fixation $(\boldsymbol{A})$, no-fixation $(\boldsymbol{B})$, and lateral fixation $(\boldsymbol{C})$ conditions. Black traces are changes in vergence during toward movements of the hand, while gray traces are changes in vergence during away movements. Note that positive and negative values correspond to an increase and decrease in vergence angle, respectively.

Table 1. Pearson's correlation coefficients between perceived size and vergence change and degrees of freedom for each participant for the three fixation conditions tested in experiment 2

\begin{tabular}{|c|c|c|c|c|c|c|}
\hline \multirow[b]{2}{*}{ Participants } & \multicolumn{2}{|c|}{ Central fixation } & \multicolumn{2}{|l|}{ No fixation } & \multicolumn{2}{|c|}{ Lateral fixation } \\
\hline & $r$ & df & $r$ & df & $r$ & $\mathrm{df}$ \\
\hline P1 & $-0.918^{*}$ & 14 & $-0.751^{*}$ & 14 & -0.200 & 14 \\
\hline P2 & $-0.929^{*}$ & 12 & $-0.860^{*}$ & 13 & -0.244 & 11 \\
\hline P3 & $-0.549^{* *}$ & 10 & $-0.476^{* *}$ & 11 & -0.314 & 12 \\
\hline P4 & $-0.881^{*}$ & 10 & $-0.617^{* *}$ & 10 & $-0.660^{*}$ & 14 \\
\hline P5 & $-0.922^{*}$ & 12 & $-0.786^{*}$ & 12 & -0.120 & 14 \\
\hline P6 & $-0.988^{*}$ & 14 & $-0.891^{*}$ & 10 & $-0.695^{*}$ & 12 \\
\hline
\end{tabular}

*Significant effects at $p_{\text {corrected }}<0.01$ (one-tailed); ** significant effects at $p_{\text {corrected }}<0.05$ (one-tailed). $P$, Participant.

given that participants still reported a size change in the afterimage when vergence was maintained on a fixed location (i.e., lateral fixation condition). To better understand the relative contribution of vergence and proprioception to the phenomenon, eye movements were recorded in experiments 2 and 3.

Experiment 2: the role of vergence and proprioception

In this experiment, we recorded eye movements to further investigate the role of vergence in the Taylor illusion. Figure 3 illustrates vergence eye movements for one representative participant. To evaluate the role played by vergence in the Taylor illusion, a correlation coefficient between size ratings and change in vergence angle was calculated for each participant (Table 1). ANOVA performed on the correlation values showed a significant effect of fixation $\left(F_{(2,10)}=14.280, p=0.001\right)$. As one would hypothesize following the results obtained from experiment 1 , this analysis confirmed that vergence greatly affected the perceived size of the afterimage. The strongest negative correlation was observed for the central fixation condition, in which both fixation light and hand movement provided concordant distance cues to the participants. However, the strength of this correlation progressively decreased from central fixation condition to nofixation and lateral fixation conditions, indicating that proprioception also contributed to the modulation of perceived size (Fig. $4 A-C)$. It is important to note that during the lateral fixation condition, small variations in vergence were detected in some trials (mean $=-0.05, \mathrm{SD}=1.49$ ). Under this condition, the correlation between vergence and perceived size was significant only in two out of six participants (Table 1). It is possible that involuntary changes in vergence invoked by arm movements
(Mon-Williams et al., 1997) were responsible for the illusion in these two participants, even though they were instructed to maintain fixation.

Post hoc pairwise comparisons on the correlation coefficients revealed significant differences between central ( mean $=-0.86$, $\mathrm{SD}=0.16)$ and no-fixation (mean $=-0.73, \mathrm{SD}=0.16$ ) conditions $\left(p_{\text {corrected }}=0.02\right)$, as well as central and lateral fixation $($ mean $=-0.37, \mathrm{SD}=0.24)$ conditions $\left(p_{\text {corrected }}=0.02\right)$. Results from the size-matching task were similar to those in experiment 1. Once again, fixation type affected the illusion $\left(F_{(2,10)}=37.211, p<0.0001\right)$. Post hoc pairwise comparisons showed significant differences between central (mean $=0.44$, $\mathrm{SD}=0.14)$ and no-fixation conditions $($ mean $=0.22, \mathrm{SD}=0.05$; $\left.p_{\text {corrected }}=0.02\right)$, central and lateral fixation conditions ( mean $=$ $\left.0.07, \mathrm{SD}=0.06 ; p_{\text {corrected }}=0.002\right)$, and lateral and no-fixation conditions $\left(p_{\text {corrected }}=0.002\right.$; Fig. $\left.4 D\right)$.

\section{Experiment 3: mismatch between vergence and proprioception}

In this experiment, we examined the relative contributions of vergence and proprioceptive cues by having participants maintain their gaze on a fixation point that moved either in the same or in the opposite direction as their moving hand. In other words, we created a discrepancy between vergence and proprioception in some trials but not in others. Table 2 reports correlation coefficients between perceived size and vergence change for congruent and incongruent conditions for each participant. Both congruent and incongruent trials showed negative correlations between changes in vergence and perceived size. However, a paired $t$ test performed on the correlation coefficients obtained in the congruent $($ mean $=-0.84, \mathrm{SD}=0.16$ ) versus incongruent (mean $=-0.76, \mathrm{SD}=0.2)$ trials revealed that the observed effects were significantly weaker when a mismatch between vergence and proprioceptive information was introduced in the incongruent trials compared with when there was no mismatch in the congruent trials $\left(t_{(5)}=-3.312, p=0.02\right.$; Fig. 5). As well, the magnitude of the illusion tested by means of a size-matching task significantly decreased during incongruent trials (congruent trials: mean $=0.45, \mathrm{SD}=0.18$; incongruent trials: mean $=0.35$, $\left.\mathrm{SD}=0.12 ; t_{(5)}=4.075, p=0.009\right)$. A $t$ test performed on vergence change for congruent trials (mean $=0.84, \mathrm{SD}=3.34$ ) versus incongruent trials ( mean $=0.36, \mathrm{SD}=2.16$ ) confirmed that vergence eye movements did not differ across the two conditions $\left(t_{(5)}=0.846, p=0.436\right)$. Together, these results provide 
further evidence that, although vergence is a major player in driving the Taylor illusion, it is not the sole source of distance information for size processing in darkness.

\section{Weighting vergence and proprioception}

Vergence and proprioceptive weights were derived from the discrepancy of the combined estimates of distance relative to the unimodal estimates of distance in the no-fixation condition of experiment 2 . This revealed that, even though the final location of the hand was fixed, distance information was more variable (i.e., less reliable) in the proprioceptive than in the vergence domain (proprioception: $\mathrm{SD}=$ 0.56 ; vergence: $\mathrm{SD}=0.27)$. Vergence weights ranged between 0.6 and 0.9 (mean $=0.8, \mathrm{SD}=0.12$ ), whereas proprioceptive weights ranged between 0.1 and $0.4($ mean $=0.2, \mathrm{SD}=0.12)$, suggesting that vergence provided more reliable distance cues for the scaling of the perceived afterimage than proprioception (Fig. 6A). Figure $6 B$ depicts observed versus predicted multimodal estimates of distance for each participant. Regression analyses were performed on predicted and observed estimates to quantify the consistency between these two values. An additional measure of consistency, the reliability coefficient (RC; Bland and Altman, 1986), was also calculated for each participant. RC expresses reliability as 2 SDs of the distribution of differences between observed and predicted estimates as a percentage of the mean of the estimates. Therefore, the smaller the RC, the more reliable the two values would be. Results from both analyses indicated that the observed and the predicted multimodal perception performances in each participant are highly correlated (Table 3 ).

\section{Discussion}

The aim of the present study was to determine the relative contribution of vergence eye movements and proprioceptive feedback to the Taylor illusion in light of claims that vergence might be the sole contributing factor driving changes in perceived afterimage size (Taylor, 1941; Mon-Williams et al., 1997). Previous studies have never actually tracked spontaneous eye movements (i.e., without a particular fixation position) during the Taylor illusion to characterize how they influence size perception. As many others have reported (Taylor, 1941; Gregory et al., 1959, Carey and Allan, 1996), the participants in our study experienced a robust Taylor illusion: the perceived size of an afterimage was smaller when the hand was positioned closer to the observer and larger when the hand was farther away. In agreement with previous observations (Carey and Allan, 1996; Bross, 2000), the results of experiment 1 showed that active and passive movements produced the same magnitude of size change in the afterimage, indicating that efference copy does not modulate the visual experience. In experiments 2 and 3, we recorded eye movements to investigate whether vergence is necessary and sufficient for the illusion to occur.
B No Fixation

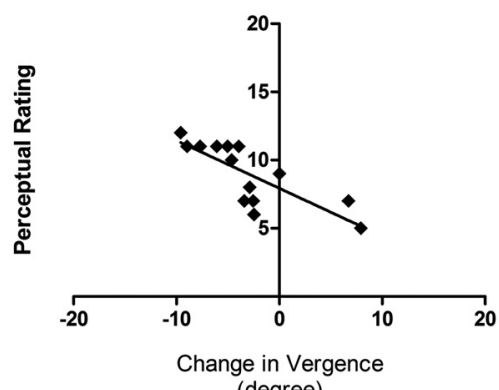

(degree)

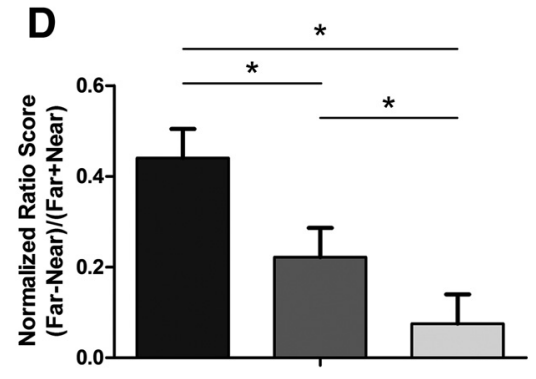

Fixation Type

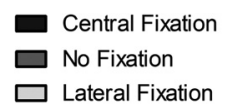

Figure 4. Results of experiment 2. $\boldsymbol{A}-\boldsymbol{C}$, Data from one representative participant demonstrate a progressive reduction of the conditions. This implies that the Taylor illusion is not solely driven by vergence signals. $\boldsymbol{D}$, Normalized effect of the Taylor illusion (six participants). Error bars indicate the within-subject 95\% confidence intervals (WSCl; based on the error term from a one-way ANOVA). Asterisks $\left({ }^{*}\right)$ denote significance after Bonferroni correction.

Table 2. Pearson's correlation coefficients between perceived size and vergence change and degrees of freedom for each participant for congruent and incongruent conditions tested in experiment 3

\begin{tabular}{llllll}
\hline & \multicolumn{2}{l}{ Congruent } & & Incongruent & \\
\cline { 2 - 4 } Participants & $r$ & $\mathrm{df}$ & & $r$ & $\mathrm{df}$ \\
\hline P1 & $-0.925^{*}$ & 13 & & $-0.840^{*}$ & 14 \\
P2 & $-0.892^{*}$ & 14 & & $-0.717^{*}$ & 13 \\
P3 & $-0.557^{* *}$ & 10 & & -0.416 & 11 \\
P4 & $-0.977^{*}$ & 14 & & $-0.956^{*}$ & 14 \\
P5 & $-0.982^{*}$ & 14 & & $-0.933^{*}$ & 13 \\
P6 & $-0.799^{*}$ & 13 & & $-0.763^{*}$ & 13 \\
\hline
\end{tabular}

*Significant effects at $p_{\text {corrected }}<0.01$ (one-tailed); ** significant effects at $p_{\text {corrected }}<0.05$ (one-tailed). $P$, Participant.

The Taylor illusion does not depend on vergence alone

The analysis of eye movements confirmed that vergence greatly affected perceived size. Indeed, it is well known that vergence is a reliable source of depth information for size constancy (Hermans, 1937; Leibowitz and Moore, 1966), and its contribution increases under reduced viewing conditions (for review, see Foley, 1980). It should also be noted that in the absence of retinal input, as in the no-fixation condition of experiment 2, the felt position of the hand was sufficient to cause a change in vergence. In these situations, hand position serves as a spatiotopic (bodycentered) stimulus to drive vergence responses in depth (Schor et al., 1992). Only a few studies have explored the role of proprioceptive feedback on vergence eye movements in darkness. Fincham (1962) found that observers could not accurately fixate their own finger in the dark and concluded that proprioception 
A

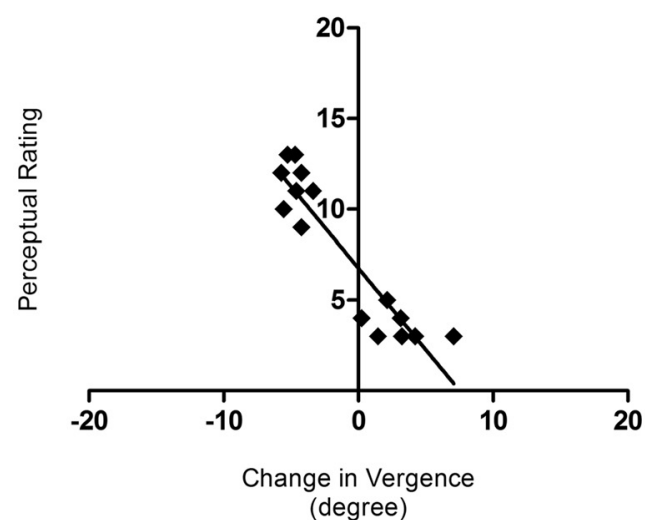

B

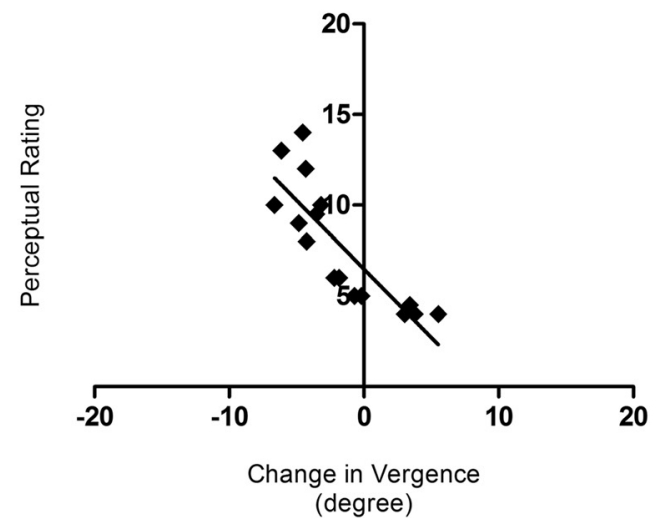

Figure 5. Results of experiment 3. $A, B$, Correlation between perceived size and vergence for one representative participant for the congruent condition, in which a fixation light was moved by the experimenter in the same direction as the observer's hand $(\boldsymbol{A})$, and the incongruent condition, in which the fixation light was moved by the experimenter in the opposite direction as the observer's hand (B). In both conditions, participants were instructed to follow the fixation light with their eyes. The slight, but significant, decrease in strength of the correlation during incongruent trials indicates that proprioceptive information is also involved in the illusion.

A

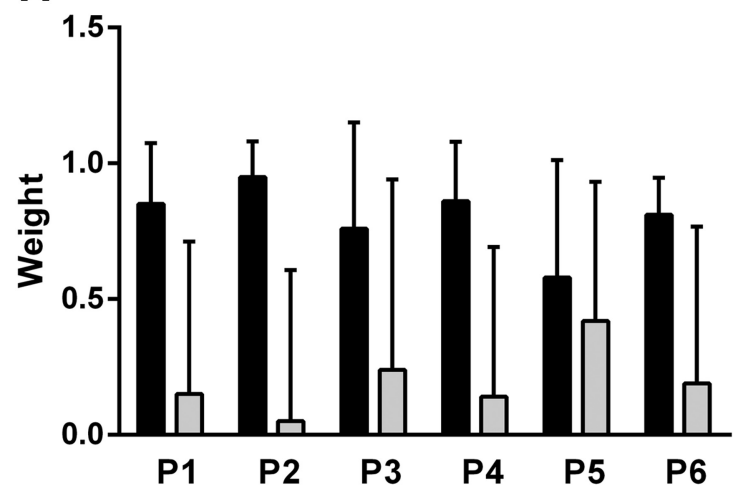

B

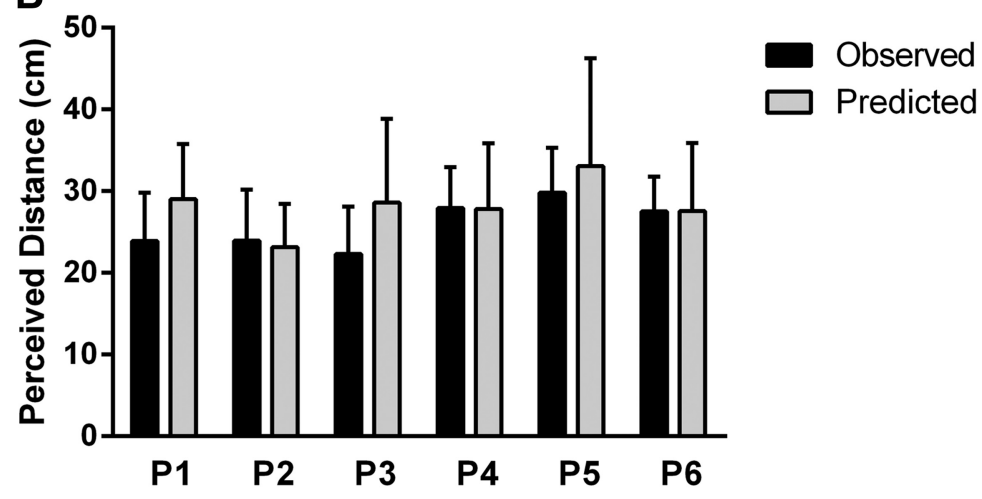

Figure 6. Relative contribution of vergence and proprioception for each participant (P) for the no-fixation condition of experiment 2.A, Vergence and proprioceptive weights. $\boldsymbol{B}$, Observed and predicted multimodal estimates of distance for far and near conditions pooled together. Error bars indicate SD.

Table 3. Coefficients of determination and reliability coefficients between observed and predicted multimodal estimates of perceived distance for each participant

\begin{tabular}{lll}
\hline Participants & $r^{2}$ & $\mathrm{RC}$ \\
\hline P1 & $0.779^{*}$ & 0.05 \\
P2 & $0.791^{* *}$ & 0.05 \\
P3 & $0.444^{* * *}$ & 0.06 \\
P4 & $0.516^{* * *}$ & 0.04 \\
P5 & $0.888^{* *}$ & 0.03 \\
P6 & $0.749^{* *}$ & 0.03 \\
\hline * & &
\end{tabular}

alone was insufficient for accurate control of vergence and accommodation. The high degree of variability in vergence angle as a function of hand position in our experiment supports this conclusion and is consistent with the idea that retinotopic (eye-centered) signals are required for fine-tuned vergence adjustments (Schor et al., 1992).

Importantly, our results showed that proprioception itself contributed to the phenomenon, as follows: the participants' reports still conformed to Emmert's law in conditions in which their eyes maintained fixation on a stationary location while the hand was moved (experiment 2). Furthermore, there was a reduction in the strength of the correlation between vergence and perceived size when a mismatch between felt hand and vergence was introduced (experiment 3). These findings suggest that the Taylor illusion is a consequence of multimodal integration of vision, vergence, and proprioceptive information from the limb. Others have come to similar conclusions. Bross (2000), for instance, obtained Taylor illusions while participants had their eyes closed after the generation of an afterimage. In this case, vergence signals might have been disrupted if the eyeballs relaxed toward their resting position (or dark vergence), which is known to be at viewing distance of $\sim 1 \mathrm{~m}$ (Owens and Leibowitz, 1976). Carey and Allan (1996) did not observe Taylor illusions when an afterimage of the nondominant hand placed in front of the dominant hand in the picture plane was induced and the dominant hand was moved. As suggested by Davies (1973), there might be a specific register between proprioceptive signals from hand position and the initial retinal input from the flash-illuminated hand for the illusion to occur. Accordingly, he demonstrated after generating an afterimage of both hands and asking the observer to 
withdraw one hand that the afterimage corresponding to the moving body part appeared to "crumble." The hypothesis of a visualproprioceptive register was directly tested by Ramsay et al. (2007) by using prism goggles that shifted the visual stimuli to the left. The authors found that the perceptual effects of the Taylor illusion were unchanged even when the prism lenses caused a location mismatch between the "visual" and the "felt" hand. According to Hogendoorn et al. (2009), prisms cannot disrupt visual-proprioceptive interactions because the higher-order bodily experiences of ownership of the visual limb are unaffected. Although we did not monitor changes in the feeling of ownership of the limb, it is worth noting that we induced afterimages of an object and not the hand. Therefore, it is quite plausible that the discrepancy induced by our lateral fixation (experiment 2) and incongruent conditions (experiment 3 ) did not disrupt such a feeling of ownership, but rather the interaction between vergence (an extraretinal signal) and proprioceptive information. The increasing conflict between modalities resulted in an increased reliance on proprioceptive signals that in turn affected perceived size.

\section{Multimodal integration in the Taylor illusion}

Our observations run contrary to a study by Mon-Williams et al. (1997) in which a fixed-vergence condition did not produce any apparent changes in perceived size. These discrepancies may relate to differences in viewing distance and effect sizes. Using the means and SDs for the reported afterimage size in their condition 2 in which a fixation point was placed at different distances by the experimenter (Mon-Williams et al., 1997, their Fig. 2), we calculated an effect size of 0.39 [estimated Cohen's $d=$ (estimated mean of size in their away condition - estimated mean of size in their toward condition)/estimated pooled SD; Cohen, 1988]. In contrast, the effect size for our congruent condition in experiment 3 was 5.07. In short, our experimental conditions created effect sizes that were much larger than those obtained by MonWilliams et al., 1997. In other words, the procedure we used to collect size judgments appeared to be more sensitive to the subtle effects of proprioception and less prone to variability than the manual estimations used in the study by Mon-Williams et al. (1997).

An exclusive vergence explanation of the Taylor illusion is further challenged by the fact that the largest changes in perceived size and the highest correlations with distance were obtained when the fixation point was on the moving hand, and thus distance cues from both the hand (proprioception) and the eyes (vergence) contributed to the effect. Other studies have suggested that size-distance scaling under reduced viewing conditions can be augmented by proprioception and haptic signals. For example, extraretinal self-motion cues produced by displacing the observer resulted in nearly perfect size constancy (Combe and Wexler, 2010). By the same token, Battaglia et al. (2010) found that size judgments were more accurate in the presence of auxiliary binocular (e.g., vergence, retinal disparity) and haptic cues, demonstrating that cross-modal distance information helps size perception.

Related to this phenomenon is the growing body of evidence on bimodal visuo-tactile coding of peri-personal space near the hand. Neurons with bimodal receptive fields that fire in response to both visual and somatosensory stimuli have been found in the putamen and in the ventral premotor cortex of the monkey (Graziano and Gross, 1993; Graziano et al., 1994). These cells have visual receptive fields that are aligned with the tactile receptive field, typically on the pericutaneous space around the hand, arm, and face. Moreover, their receptive fields move with the body part, not with the eyes, and the strength of their response diminishes as the distance from the body part increases. Interestingly, these neurons code for the position of the unseen arm (Graziano, 1999). The properties of bimodal neurons suggest that visual processing can benefit from proprioceptive information. This hypothesis has been supported by behavioral, neuropsychologi$\mathrm{cal}$, and neuroimaging research. For example, it has been demonstrated that placing the hand near the visual stimulus facilitates its detection by patients with visual extinction (di Pellegrino and Frassinetti, 2000) as well as by healthy individuals (Reed et al., 2006). Also, the accuracy of reaching movements in darkness seems to be improved by information about hand location (Monaco et al., 2010). Remarkably, Brown et al. (2008) described an improvement in unconscious residual vision in cortically blind patients when the hand was placed close to a target presented in the blind field. Recent fMRI studies in humans indicate the parietal cortex as a possible neural locus for bimodal cells (Makin et al., 2007). The parietal cortex, which is typically involved in size computation during reaching and grasping (Culham et al., 2003; Cavina-Pratesi et al., 2007; Chouinard et al., 2009), would mediate the integration of visual and somatosensory information within peri-personal space for visuomotor control. The neural circuits underlying size-constancy computations, and their relationship to the Taylor illusion, remain unknown, although there is evidence that size constancy might be at work even in early visual areas. An fMRI study (Sperandio et al., 2012) showed that activation in the primary visual cortex (V1) was modulated by the perceived rather than the retinal size of an afterimage projected at different viewing distances, demonstrating that retinal image and distance information can be processed together in V1. Perhaps the same modulation can be observed during the Taylor illusion as a result of top-down feedback from parietal regions that contain bimodal cells.

\section{Closing remarks}

Results of the present experiments, along with earlier work, lead to the conclusion that hand-centered representations of space influence size-constancy mechanisms, particularly under conditions in which vision cannot override information from other sensory modalities.

\section{References}

Alais D, Burr D (2004) The ventriloquist effect results from near-optimal bimodal integration. Curr Biol 14:257-262. CrossRef Medline

Andrews DP (1964) Error-correcting perceptual mechanisms. Q J Exp Psychol 16:105-115.

Battaglia PW, Di Luca M, Ernst MO, Schrater PR, Machulla T, Kersten D (2010) Within- and cross-modal distance information disambiguate visual size-change perception. PLoS Comput Biol 6:e1000697. CrossRef Medline

Bland JM, Altman DG (1986) Statistical methods for assessing agreement between two methods of clinical measurement. Lancet 1:307-310. Medline

Bross M (2000) Emmert's law in the dark: active and passive proprioceptive effects on positive visual afterimages. Perception 29:1385-1391. CrossRef Medline

Brown LE, Kroliczak G, Demonet JF, Goodale MA (2008) A hand in blindsight: hand placement near target improves size perception in the blind visual field. Neuropsychologia 46:786-802. CrossRef Medline

Carey DP, Allan K (1996) A motor signal and "visual” size perception. Exp Brain Res 110:482-486. Medline

Cavina-Pratesi C, Goodale MA, Culham JC (2007) FMRI reveals a dissociation between grasping and perceiving the size of real 3D objects. PLoS One 2:e424. CrossRef Medline

Chouinard PA, Large ME, Chang EC, Goodale MA (2009) Dissociable neural mechanisms for determining the perceived heaviness of objects and 
the predicted weight of objects during lifting: an fMRI investigation of the size-weight illusion. Neuroimage 44:200-212. CrossRef Medline

Chouinard PA, Noulty WA, Sperandio I, Landry O (2013) Global processing during the Müller-Lyer illusion is distinctively affected by the degree of autistic traits in the typical population. Exp Brain Res 230:219-231. CrossRef Medline

Cohen J (1988) Statistical power analysis for the behavioral sciences. Hillsdale, NJ: Erlbaum.

Combe E, Wexler M (2010) Observer movement and size constancy. Psychol Sci 21:667-675. CrossRef Medline

Culham JC, Danckert SL, DeSouza JF, Gati JS, Menon RS, Goodale MA (2003) Visually guided grasping produces fMRI activation in dorsal but not ventral stream brain areas. Exp Brain Res 153:180-189. CrossRef Medline

Davies P (1973) Effects of movements upon the appearance and duration of a prolonged visual afterimage: 1 . Changes arising from the movement of a portion of the body incorporated in the afterimaged scene. Perception 2:147-153. CrossRef

di Pellegrino G, Frassinetti F (2000) Direct evidence from parietal extinction of enhancement of visual attention near a visible hand. Curr Biol 10:1475-1477. CrossRef Medline

Emmert E (1881) Grössenverhältnisse der Nachbilder. Klin Monatbl Augenheilkd 19:443-450.

Ernst MO, Banks MS (2002) Humans integrate visual and haptic information in a statistically optimal fashion. Nature 415:429-433. CrossRef Medline

Fincham EF (1962) Accommodation and convergence in the absence of retinal images. Vision Res 1:425-440. CrossRef

Foley JM (1980) Binocular distance perception. Psychol Rev 87:411-434. CrossRef Medline

Frey J, Ringach DL (2011) Binocular eye movements evoked by self-induced motion parallax. J Neurosci 31:17069-17073. CrossRef Medline

Gepshtein S, Banks MS (2003) Viewing geometry determines how vision and haptics combine in size perception. Curr Biol 13:483-488. CrossRef Medline

Graziano MS (1999) Where is my arm? The relative role of vision and proprioception in the neuronal representation of limb position. Proc Natl Acad Sci U S A 96:10418-10421. CrossRef Medline

Graziano MS, Gross CG (1993) A bimodal map of space: somatosensory receptive fields in the macaque putamen with corresponding visual receptive fields. Exp Brain Res 97:96-109. Medline

Graziano MS, Yap GS, Gross CG (1994) Coding of visual space by premotor neurons. Science 266:1054-1057. CrossRef Medline

Gregory RL, Wallace JG, Campbell FW (1959) Changes in size and shape of visual afterimages observed in complete darkness during changes of position in space. Q J Exp Psychol 11:54-55. CrossRef
Hermans TG (1937) Visual size constancy as a function of convergence. J Exp Psychol 21:307-324.

Hogendoorn H, Kammers MP, Carlson TA, Verstraten FA (2009) Being in the dark about your hand: resolution of visuo-proprioceptive conflict by disowning visible limbs. Neuropsychologia 47:2698-2703. CrossRef Medline

Landy MS, Maloney LT, Johnston EB, Young M (1995) Measurement and modeling of depth cue combination: in defense of weak fusion. Vision Res 35:389-412. CrossRef Medline

Leibowitz H, Moore D (1966) Role of changes in accommodation and convergence in the perception of size. J Opt Soc Am 56:1120-1123. CrossRef Medline

Loftus GR, Masson MEJ (1994) Using confidence intervals in withinsubject designs. Psychon Bull Rev 1:476-490. CrossRef

Makin TR, Holmes NP, Zohary E (2007) Is that near my hand? Multisensory representation of peripersonal space in human intraparietal sulcus. J Neurosci 27:731-740. CrossRef Medline

Monaco S, Króliczak G, Quinlan DJ, Fattori P, Galletti C, Goodale MA, Culham JC (2010) Contribution of visual and proprioceptive information to the precision of reaching movements. Exp Brain Res 202:15-32. CrossRef Medline

Mon-Williams M, Tresilian JR, Plooy A, Wann JP, Broerse J (1997) Looking at the task in hand: vergence eye movements and perceived size. Exp Brain Res 117:501-506. CrossRef Medline

Morgan MJ (1992) On the scaling of size judgments orientational cues. Vis Res 32:1433-1445. CrossRef Medline

Oldfield RC (1971) The assessment and analysis of handedness: the Edinburgh inventory. Neuropsychologia 9:97-113. Medline

Owens DA, Leibowitz HW (1976) Ocular adjustment in darkness and the specific distance tendency. Percept Psychophys 20:2-9. CrossRef

Ramsay AI, Carey DP, Jackson SR (2007) Visual-proprioceptive mismatch and the Taylor illusion. Exp Brain Res 176:173-181. CrossRef Medline

Reed CL, Grubb JD, Steele C (2006) Grasping attention: the effects of hand proximity on visual covert orienting. J Exp Psychol Hum Percept Perform 32:166-177. CrossRef

Schor CM, Alexander J, Cormack L, Stevenson S (1992) Negative feedback control model of proximal convergence and accommodation. Ophthalmic Physiol Opt 12:307-318. CrossRef Medline

Sperandio I, Chouinard PA, Goodale MA (2012) Retinotopic activity in V1 reflects the perceived not the retinal size of an after-image. Nat Neurosci 15:540-542. CrossRef Medline

Taylor FV (1941) Change in size of the afterimage induced in total darkness. J Exp Psychol 29:75-80. CrossRef

Tresilian JR, Mon-Williams M (2000) Getting the measure of vergence weight in nearness perception. Exp Brain Res 132:362-368. CrossRef Medline 